\title{
Sum rules and cutoff effects in Wilson lattice QCD
}

\author{
Harvey B. Meyer \\ Deutsches Elektronen-Synchrotron DESY, Platanenallee 6, D-15738 Zeuthen, Germany \\ Received 13 September 2006; accepted 18 October 2006 \\ Available online 2 November 2006
}

\begin{abstract}
We use the transfer matrix formalism to derive non-perturbative sum rules in Wilson's lattice QCD with $N_{\mathrm{f}}$ flavours of quarks. The discretization errors on these identities are treated in detail. As an application, it is shown how the sum rules can be exploited to give improved estimates of the continuum spectrum and static potential.

(c) 2006 Elsevier B.V. All rights reserved.
\end{abstract}

\section{Introduction}

Although relatively old, the subject of lattice sum rules [1,2] in $d=4 \mathrm{SU}(N)$ gauge theories has received only sporadic attention since it was initiated by Michael [3]. In essence, these identities relate the derivative with respect to a bare lattice parameter of a physical quantity to a higher order correlation function. A prototype sum rule states that the $\beta$-dependence of an energy level is determined by the expectation value of the Lagrangian on that state.

In Monte Carlo simulations, sum rules were first applied as a cross-check in form factor calculations at zero-momentum transfer [4], and in measurements of the flux-tube profile in the static $Q \bar{Q}$ system [5]. Later they were used to determine lattice beta-functions non-perturbatively $[6,7]$. On the theory side, they have also led to some insight into the relative contribution to the mass of a glueball of the component $T_{00}$ of the traceless energy-momentum tensor and of the trace anomaly [8].

Here we shall generalize the sum rules to the case where $N_{\mathrm{f}}$ flavours of Wilson quarks are present. We do so by invoking the transfer matrix, in our view the most elegant approach. It also makes it clear that the identities derived do not depend on the temporal boundary conditions or

E-mail addresses: harvey.meyer@desy.de, meyerh@mit.edu (H.B. Meyer). 
on which phase the system is in-be it a finite-temperature phase, a parity-broken phase or other. Let us recall the Wilson action, which has two parameters $(\beta, \kappa)$ :

$$
\begin{aligned}
S_{\mathrm{g}}= & \beta \sum_{p} \square_{p}, \quad \square_{p}=\frac{1}{N} \operatorname{Re} \operatorname{Tr}\{1-U(p)\}, \\
S_{\mathrm{f}}= & \sum_{x} \bar{\psi}(x) \psi(x) \\
& -\kappa \sum_{\mu}\left[\bar{\psi}(x) U_{\mu}(x)\left(1-\gamma_{\mu}\right) \psi(x+a \hat{\mu})+\bar{\psi}(x+a \hat{\mu}) U_{\mu}^{\dagger}(x)\left(1+\gamma_{\mu}\right) \psi(x)\right],
\end{aligned}
$$

where in Eq. (1) the sum extends over all unoriented plaquettes.

It is well known that cutoff effects are of $\mathrm{O}(a)$ in Wilson's formulation. In recent times, most large-scale numerical calculations have made use of the $\mathrm{O}(a)$ improvement program [10] to reduce these effects (with some notable exceptions [11,12]). We present an idea to exploit lattice sum rules in order to extract additional information on the lattice spacing dependence of the spectrum, and further how its lattice artifacts can be reduced.

The idea is also applicable to the energy levels in the presence of a $Q \bar{Q}$ pair, where one is typically interested in their derivatives with respect to the static quark separation. The static force, and the effective central charge in the pure gauge case, are affected by discretization errors which can be large compared to the statistical precision one is able to achieve.

In Section 2 we rederive some of the known results in the pure gauge theory, where particular attention is paid to discretization errors. In Section 3 the sum rules are derived for full QCD. Concrete applications are proposed in both sections. We make some concluding remarks in Section 4.

\section{Transfer matrix and sum rules in the pure gauge theory}

We start with a brief reminder on the transfer formalism and how sum rules are derived in the pure gauge theory. Some familiarity with the lattice regularization is assumed. For dimensionful quantities in lattice units we use the notation $L=\breve{L} a$, etc. We shall assume periodic boundary conditions in the spatial directions and the non-degeneracy of the vacuum state.

If $V$ is the set of spatial link variables in a time-slice, let $\Phi[V] \in \mathcal{H}_{G}$ be a square-integrable wave function with respect to the Haar measure of the gauge group. The transfer matrix $\mathbf{T}_{\beta}$ acts as follows [13-15]:

$$
\left(\mathbf{T}_{\beta} \Phi\right)[V]=\int \prod_{\mathbf{x}} \prod_{k=1}^{3} \mathrm{~d} V_{k}^{\prime}(\mathbf{x}) K_{\beta}\left[V, V^{\prime}\right] \Phi\left[V^{\prime}\right],
$$

with kernel

$$
\begin{aligned}
& K_{\beta}\left[V, V^{\prime}\right]=\int \prod_{\mathbf{x}} \mathrm{d} W(\mathbf{x}) \exp \left(-\beta \Delta S\left[V, W, V^{\prime}\right]\right), \\
& \Delta S\left[V, W, V^{\prime}\right]=\sum_{k} \square_{0 k}\left[V, W, V^{\prime}\right]+\frac{1}{2} \sum_{k<l}\left(\square_{k l}[V]+\square_{k l}\left[V^{\prime}\right]\right), \\
& \square_{0 k}\left[V, W, V^{\prime}\right] \equiv \frac{1}{N} \sum_{\mathbf{x}} \operatorname{Re} \operatorname{Tr}\left\{1-V_{k}^{\prime}(\mathbf{x}) W(\mathbf{x}+a \hat{k}) V_{k}(\mathbf{x})^{-1} W(\mathbf{x})^{-1}\right\} .
\end{aligned}
$$


In words, $\Delta S$ is the restriction of the action to the region of space-time between $x_{0}$ and $x_{0}+a$, with a weight of $1 / 2$ given to the terms living on the boundary. When the full gauge system is defined with periodic boundary conditions in the time direction, the partition function is given by $\mathcal{Z}(\beta)=\operatorname{Tr}\left\{\mathbf{T}_{\beta} \breve{L}_{0}\right\}$.

We define the magnetic-plaquette operator $\hat{\square}_{k l}$ to act as in Eq. (3) with $K_{\beta}$ replaced by

$$
K_{k l}\left[V, V^{\prime}\right]=\delta\left(V-V^{\prime}\right) \square_{k l}[V] .
$$

Similarly, the kernel corresponding to the electric plaquette operator $\hat{\square}_{0 k}$ is defined as

$$
K_{0 k}\left[V, V^{\prime}\right]=\lambda_{\Omega}^{-1} \int \prod_{\mathbf{x}} \mathrm{d} W(\mathbf{x}) \exp \left(-\beta \Delta S\left[V, W, V^{\prime}\right]\right) \square_{0 k}\left[V, W, V^{\prime}\right],
$$

where $\mathbf{T}_{\beta}|\Omega\rangle=\lambda_{\Omega}|\Omega\rangle$. The operators $\hat{\square}_{k l}$ and $\hat{\square}_{0 k}$ are such that their vacuum expectation values are equal to the Euclidean expectation values of the spatial and temporal plaquettes with periodic boundary conditions in the time direction, the time extent being sent to infinity. Then, if $\mathbf{T}_{\beta}|\Phi\rangle=\lambda|\Phi\rangle$,

$$
\begin{aligned}
-\partial_{\beta} \lambda(\beta) & =-\partial_{\beta}\left\langle\Phi\left|\mathbf{T}_{\beta}\right| \Phi\right\rangle=-\left\langle\Phi\left|\partial_{\beta} \mathbf{T}_{\beta}\right| \Phi\right\rangle \\
& =\lambda\left\langle\Phi\left|\sum_{k<l} \hat{\square}_{k l}\right| \Phi\right\rangle+\lambda_{\Omega}\left\langle\Phi\left|\sum_{k} \hat{\square}_{0 k}\right| \Phi\right\rangle .
\end{aligned}
$$

Since $|\Phi\rangle$ can be any eigenstate of the transfer matrix, we can choose it to be successively the vacuum and a glueball state. The spatial volume is assumed to be large enough for the finitevolume effects on the mass gap to be negligible. Subtracting the two corresponding rules gives

$$
\frac{\mathrm{d} \breve{M}}{\mathrm{~d} \beta}=\sum_{k<l}\left\langle\Phi\left|\hat{\square}_{k l}\right| \Phi\right\rangle-\left\langle\Omega\left|\hat{\square}_{k l}\right| \Omega\right\rangle+\sum_{k} e^{\breve{M}}\left\langle\Phi\left|\hat{\square}_{0 k}\right| \Phi\right\rangle-\left\langle\Omega\left|\hat{\square}_{0 k}\right| \Omega\right\rangle .
$$

Here $\breve{M}=-\log \lambda / \lambda_{\Omega}$ is the mass of the glueball in lattice units.

Following [1], we can use the fact that $d / d a(\breve{M} / a)=0$ in the continuum limit to conclude

$$
\begin{aligned}
\breve{M}= & \frac{\mathrm{d} \beta}{\mathrm{d} \log a}\left\{\sum_{k<l}\left\langle\Phi\left|\hat{\square}_{k l}\right| \Phi\right\rangle-\left\langle\Omega\left|\hat{\square}_{k l}\right| \Omega\right\rangle+\sum_{k} e^{\breve{M}}\left\langle\Phi\left|\hat{\square}_{0 k}\right| \Phi\right\rangle-\left\langle\Omega\left|\hat{\square}_{0 k}\right| \Omega\right\rangle\right\} \\
& \times\left[1+\mathrm{O}\left(a^{2}\right)\right] .
\end{aligned}
$$

To translate the matrix elements appearing on the right-hand side into Euclidean correlators, for $\Phi$ the lightest state in its symmetry channel one may choose a zero-momentum linear combination $\phi\left(x_{0}\right)$ of magnetic loops in that channel, and compute the correlator

$$
\frac{\left\langle\bar{\phi}\left(x_{0}+a\right) \frac{1}{2}\left(\sum_{k<l} P_{k l}\left(x_{0} / 2\right)+P_{k l}\left(x_{0} / 2+a\right)\right) \bar{\phi}(0)\right\rangle}{\left\langle\bar{\phi}\left(x_{0}+a\right) \bar{\phi}(0)\right\rangle} \stackrel{x_{0} \rightarrow \infty}{\rightarrow} \sum_{k<l}\left\langle\Phi\left|\hat{\square}_{k l}\right| \Phi\right\rangle,
$$

where $\bar{\phi}\left(x_{0}\right)=\phi\left(x_{0}\right)-\langle\phi\rangle$ (take $x_{0} / a$ even). And similarly

$$
\frac{\left\langle\bar{\phi}\left(x_{0}+a\right) \sum_{k} P_{0 k}\left(x_{0} / 2\right) \bar{\phi}(0)\right\rangle}{\left\langle\bar{\phi}\left(x_{0}+a\right) \bar{\phi}(0)\right\rangle} \stackrel{x_{0} \rightarrow \infty}{\rightarrow} e^{\breve{M}(\beta)} \sum_{k}\left\langle\Phi\left|\hat{\square}_{0 k}\right| \Phi\right\rangle .
$$




\subsection{Improved estimators of the continuum spectrum}

We use the shorthand notation $\frac{\mathrm{d} \breve{M}}{\mathrm{~d} \beta}=\square_{\Omega}^{\Phi}$ for Eq. (10) from now on. In view of taking the continuum limit, we use a reference length $\ell_{0}$ which sets the scale of the theory. Typical examples are $\sigma^{-1 / 2}$, where $\sigma$ is the string tension and $r_{0}$, the Sommer reference scale [19]. We assume that for every $\beta, \breve{\ell}_{0} \equiv \ell_{0} / a$ can be determined, so that $\beta\left(\breve{\ell}_{0}\right)$, which is assumed to be monotonic in the range of interest, and

$$
\frac{\mathrm{d} \beta}{\mathrm{d} \log a} \equiv-\frac{\mathrm{d} \beta\left(\breve{\ell}_{0}\right)}{\mathrm{d} \log \breve{\ell}_{0}}
$$

are well defined. For the case $\ell_{0}=r_{0}, \breve{\ell}_{0}(\beta)$ is known with one percent precision or better [18]. Thus on a line of constant physics, a function of $\beta$ can just as well be regarded as a function of $\breve{\ell}_{0}$, and vice versa.

The dimensionless quantity $z \equiv \ell_{0} M$ has a continuum limit, and we can express its derivative with respect to the lattice spacing exactly:

$$
\frac{\mathrm{d} z}{\mathrm{~d} \breve{\ell}_{0}}=\breve{M}-\frac{\mathrm{d} \beta}{\mathrm{d} \log a} \square_{\Omega}^{\Phi} .
$$

This information on the slope can be included in the continuum extrapolation if data at several lattice spacings is available. Even if not, it can be used to provide an improved estimate of the continuum limit of $z$ if we assume a particular form of the discretization errors. In practice $z$ is usually observed to approach the continuum with $\mathrm{O}\left(a^{2}\right)$ corrections within statistical errors; such corrections can be removed according to

$$
z_{\text {impr }} \equiv z\left(\breve{\ell}_{0}\right)+\frac{1}{2} \breve{\ell}_{0} \frac{\mathrm{d} z}{\mathrm{~d} \breve{\ell}_{0}}=\frac{3}{2} z\left(\breve{\ell}_{0}\right)-\frac{\breve{\ell}_{0}}{2} \frac{\mathrm{d} \beta}{\mathrm{d} \log a} \square_{\Omega}^{\Phi} .
$$

In what sense is this an improved estimate of the continuum limit? Suppose the true analytic form of $z$ is $[9,21]$

$$
z\left(\breve{\ell}_{0}\right)=z_{\text {cont }}+\frac{1}{\breve{\ell}_{0}^{2}} \sum_{n=0}^{N} c_{n} \log ^{n}\left\{1 / \breve{\ell}_{0}\right\}+\mathrm{O}\left(1 / \breve{\ell}_{0}^{4}\right) .
$$

Then

$$
z_{\text {impr }}=z_{\text {cont }}-\frac{1}{2 \breve{\ell}_{0}^{2}} \sum_{n=0}^{N-1}(n+1) c_{n+1} \log ^{n}\left\{1 / \breve{\ell}_{0}\right\}+\mathrm{O}\left(1 / \breve{\ell}_{0}^{4}\right) .
$$

Since the series of logarithms is asymptotic, if $N=1$ yields the best accuracy at the lattice spacing one is working at, then $\left|c_{0}\right| \gg\left|c_{1} \log \left\{1 / \breve{\ell}_{0}\right\}\right| \gg\left|c_{1}\right|$, so that $z_{\text {impr }}$ has reduced discretization errors, although it is not a full $\mathrm{O}\left(a^{2}\right)$ improvement. Note that it is essential in (16) to use the quantity $\ell_{0}$ that appears elsewhere in the formula to define $\frac{\mathrm{d} \beta}{\mathrm{d} \log a}$, as in Eq. (14).

\subsection{States depending on an external physical scale}

We now consider an eigenstate of a transfer matrix which, in addition of depending on $\beta$, also depends on an external physical scale $s$, and the latter can take only integer multiples of some 
quantum $\Delta s$ :

$$
s=n \Delta s, \quad n \in \mathbf{N} .
$$

Examples are zero-momentum states in finite volume (where $s=L, \Delta s=a$ ), but also largevolume states with non-vanishing momentum $(s=p, \Delta s=2 \pi / L)$. The sum rule then reads

$$
\frac{\partial \breve{E}}{\partial \beta}(n, \beta)=\square_{\Omega}^{\Phi(n)} \equiv \square_{\Omega}^{n} .
$$

We shall make use of the standard notation for discrete differences

$$
\begin{aligned}
& \partial_{n} f(n)=f(n+1)-f(n), \quad \partial_{n}^{*} f(n)=f(n)-f(n-1), \\
& \tilde{\partial}_{n} f(n)=\frac{1}{2}(f(n+1)-f(n-1)), \\
& \Delta_{n} f(n)=f(n+1)-2 f(n)+f(n-1) .
\end{aligned}
$$

Let us consider again the quantity $z=\breve{\ell}_{0} \breve{E}$, which has a continuum limit. For definiteness take the case of a zero-momentum state in finite volume, so that $L=n a$. For a given couple $(n, \beta)$, we choose an auxiliary $n^{\prime}$ close to but different from $n$; there is a $\beta^{\prime}$ such that $n^{\prime} / n=\breve{\ell}_{0}\left(\beta^{\prime}\right) / \breve{\ell}_{0}(\beta)$; that is, the box sizes are matched in physical units. Then we can write

$$
\begin{aligned}
\breve{\ell}_{0} \breve{E}(n, \beta)= & \breve{\ell}_{0}\left(\beta^{\prime}\right) \breve{E}\left(n^{\prime}, \beta^{\prime}\right)\left[1+\mathrm{O}\left(a^{2}\right)\right] \\
= & \breve{\ell}_{0} \frac{n^{\prime}}{n}\left[\breve{E}\left(n^{\prime}, \beta\right)+\frac{\mathrm{d} \beta}{\mathrm{d} \log a} \log \left\{n / n^{\prime}\right\} \square_{\Omega}^{n^{\prime}}(\beta)+\frac{1}{2} \log ^{2}\left\{n / n^{\prime}\right\} f\left(n^{\prime}, \beta\right)\right] \\
& \times\left[1+\mathrm{O}\left(a^{2}\right)\right],
\end{aligned}
$$

where $f\left(n^{\prime}, \beta\right)=\frac{\mathrm{d}^{2} \beta}{\mathrm{d}(\log a)^{2}} \partial_{\beta} \breve{E}\left(n^{\prime}, \beta\right)+\partial_{\beta}^{2} E\left(n^{\prime} \beta\right)\left(\frac{\mathrm{d} \beta}{\mathrm{d} \log a}\right)^{2}$. This second order term is necessary to eliminate $\mathrm{O}(a)$ cutoff effects. We now choose $n^{\prime}=n-1$ and then shift $n \rightarrow n+1$, obtaining equation (a); secondly we choose $n^{\prime}=n+1$ and then shift $n \rightarrow n-1$, obtaining equation (b). Note that the function $f(n, \beta)$ is evaluated at the same arguments in (a) and (b). Therefore we can eliminate that term by taking the linear combination $\log ^{2}(1-1 / n) \cdot(a)-\log ^{2}(1+1 / n) \cdot(b)$. This results in

$$
\frac{\mathrm{d} \beta}{\mathrm{d} \log a} \square_{\Omega}^{n}\left[1+\mathrm{O}\left(a^{2}\right)\right]=\breve{E}(n)+n \tilde{\partial}_{n} \breve{E}(n),
$$

where we dropped the $\beta$ dependence and used the freedom to trade one discretization scheme for another that is equivalent to $\mathrm{O}\left(a^{2}\right)$. This is one of the Michael-Rothe sum rules [1,2]. We have however kept track of the discretization errors carefully so as not to introduce $\mathrm{O}(a)$ effects. This sum rule allows one to extract the derivative of the torelon energy with respect to its length without having to perform an independent simulation. In particular the effective central charge can be computed via

$$
c_{\mathrm{eff}}\left(L=n a, \breve{\ell}_{0}\right)=\frac{n}{2} \frac{\mathrm{d} \beta}{\mathrm{d} \log a} \square_{\Omega}^{n}-n \breve{E}(n)
$$




\subsection{The static potential case}

The sum rule (20) also holds if static charges are inserted at points $\mathbf{0}$ and $\mathbf{x}, \mathbf{x}=r \hat{k}$. Indeed the kernel of the transfer matrix then projects onto states $\Phi_{\alpha \beta} \ldots$ which, under a gauge transformation $U \rightarrow U^{\Lambda}$, transform with $\Lambda(\mathbf{0})$ and $\Lambda(\mathbf{x})$ according to the representation of these charges. For a fundamental-antifundamental pair, we have explicitly:

$$
\Phi_{\alpha \beta}\left[U^{\Lambda}\right]=\left(\Lambda(\mathbf{0})_{\alpha \gamma}\right)^{*} \Lambda(\mathbf{x})_{\beta \delta} \Phi_{\gamma \delta}[U] .
$$

The transfer matrix kernel is

$$
K_{\mathbf{N}^{*} \otimes \mathbf{N}}^{\alpha \beta \gamma \delta}=\int \prod_{\mathbf{x} x} \mathrm{~d} W(\mathbf{x}) \exp \left(-\beta \Delta S\left[V, W, V^{\prime}\right]\right)\left(W(\mathbf{0})_{\alpha \beta}\right)^{*} W(\mathbf{x})_{\gamma \delta}
$$

and the composition rule is

$$
\left(\mathbf{T}_{\mathbf{N}^{*} \otimes \mathbf{N}}^{2}\right)^{\alpha \beta \gamma \delta}=\left(\mathbf{T}_{\mathbf{N}^{*} \otimes \mathbf{N}}\right)^{\alpha \lambda \gamma \epsilon}\left(\mathbf{T}_{\mathbf{N}^{*} \otimes \mathbf{N}}\right)^{\lambda \beta \epsilon \delta} .
$$

Thus the partition function in the presence of the static charges is

$$
\mathcal{Z}_{\mathbf{N}^{*} \otimes \mathbf{N}}=\frac{1}{N^{2}} \sum_{\alpha, \gamma} \operatorname{Tr}\left\{\left(\mathbf{T}_{\mathbf{N}^{*} \otimes \mathbf{N}}^{\breve{L}_{0}}\right)^{\alpha \alpha \gamma \gamma}\right\} .
$$

In particular, the Polyakov loop correlator evaluates to [16] $\left\langle P(\mathbf{0})^{*} P(\mathbf{x})\right\rangle=\mathcal{Z}_{\mathbf{N}^{*} \otimes \mathbf{N}} / \mathcal{Z}$.

The energies at two different separations $r$ must be subtracted in order to remove the divergent self-energy of the static quarks. The quantity $\breve{\ell}_{0}^{2}(\beta) \partial_{n} \breve{E}(n, \beta)$ has a continuum limit. We define $\bar{n}=n+\frac{1}{2}+\mathrm{O}\left(a^{2}\right)$ where the $\mathrm{O}\left(a^{2}\right)$ need not be specified presently, and choose two integers $n, n^{\prime} \gg 1,\left|n-n^{\prime}\right|=\mathrm{O}(1)$ and a particular value of $\beta$. Now there exists an auxiliary $\beta^{\prime}$ such that

$$
\bar{n} a(\beta)=\bar{n}^{\prime} a\left(\beta^{\prime}\right), \quad \text { i.e. } \quad \bar{n} \breve{\ell}_{0}\left(\beta^{\prime}\right)=\bar{n}^{\prime} \breve{\ell}_{0}(\beta) .
$$

We can write $\breve{\ell}_{0}^{2}(\beta) \partial_{n} \breve{E}(n, \beta)=\breve{\ell}_{0}^{2}\left(\beta^{\prime}\right) \partial_{n^{\prime}} \breve{E}\left(n^{\prime}, \beta^{\prime}\right)\left[1+\mathrm{O}\left(a^{2}\right)\right]$ and then Taylor-expand in $\beta$ to obtain in the same way as in the previous section

$$
n \Delta_{n} \breve{E}(n)+2 \tilde{\partial}_{n} \breve{E}(n)=\frac{1}{2} \frac{\mathrm{d} \beta}{\mathrm{d} \log a} \square_{n-1}^{n+1}\left[1+\mathrm{O}\left(a^{2}\right)\right] .
$$

We use the notation $\square_{n-1}^{n+1} \equiv \square_{\Omega}^{n+1}-\square_{\Omega}^{n-1}$. Again a symmetric finite-difference scheme is necessary and sufficient to remove the $\mathrm{O}(a)$ discretization errors.

\subsubsection{An improved estimate of $r_{0}^{2} F(r)$}

We now show how one can also use the lattice sum rule to reduce cutoff effects on the static force. Discretization errors are by far the dominant source of uncertainty on this quantity [16]. Set

$$
\ell_{0} \doteq r_{0} \quad \text { and } \quad z\left(\xi, \breve{r}_{0}\right) \doteq \breve{r}_{0}^{2} F\left(r=\xi r_{0}, \breve{r}_{0}\right) \text {. }
$$

A direct measurement of the force is

$$
F\left(\bar{n} a, \breve{r}_{0}\right)=\partial_{n} E\left(n, \breve{r}_{0}\right) .
$$

This defines the static force at a discrete set of points. To define it for all distances, an interpolation formula between neighbouring points must be used. Which formula is a matter of choice (different definitions of the force at finite lattice spacing will then differ by $\mathrm{O}\left(a^{2}\right)$ ). 
For illustration we choose a linear interpolation between the two nearest direct measurements of the static force:

$$
z\left(\xi, \breve{r}_{0}\right)=\frac{\breve{r}_{0}^{2}}{\bar{n}-\overline{n-1}}\left[\left(\xi \breve{r}_{0}-\overline{n-1}\right) \partial_{n} \breve{E}\left(n, \breve{r}_{0}\right)+\left(\bar{n}-\xi \breve{r}_{0}\right) \partial_{n}^{*} \breve{E}\left(n, \breve{r}_{0}\right)\right] .
$$

With this precise definition and Eq. (20), $\partial z / \partial \breve{r}_{0}$ can be evaluated at fixed $\xi$ exactly. An improved estimate of the continuum $z$ reads

$$
z_{\text {impr }}\left(\xi, \breve{r}_{0}\right)=z\left(\xi, \breve{r}_{0}\right)+\frac{1}{2} \breve{r}_{0} \frac{\partial z}{\partial \breve{r}_{0}},
$$

which is improved in the same sense as the glueball mass in Section 2.1. Explicitly, we get

$$
\begin{aligned}
z_{\operatorname{impr}}\left(\xi, \breve{r}_{0}\right)= & 2 z\left(\xi, \breve{r}_{0}\right)+\frac{\frac{1}{2} \breve{r}_{0}^{2}}{\bar{n}-\overline{n-1}} \\
& \times\left[\xi \breve{r}_{0} \Delta_{n} E\left(n, \breve{r}_{0}\right)-\frac{\mathrm{d} \beta}{\mathrm{d} \log a}\left(\left(\xi \breve{r}_{0}-\overline{n-1}\right) \square_{n}^{n+1}+\left(\bar{n}-\xi \breve{r}_{0}\right) \square_{n-1}^{n}\right)\right] .
\end{aligned}
$$

It is important to realize that the improvement is not merely a higher order difference scheme for the static force, rather it contains non-perturbative information about its lattice spacing dependence. Formula (34) may be numerically useful since all terms on the right-hand side can be evaluated in the same simulation. Note that the idea is applicable to more complicated interpolation schemes for any particular definition of $\bar{n}$, and also to the effective central charge $c_{\text {eff }}(r)=-\frac{1}{2} r^{3} \mathrm{~d}^{2} V / \mathrm{d} r^{2}$.

\subsection{Anisotropic couplings}

Sum rules in the pure gauge theory derived by varying the lattice spacings in the four spacetime directions independently around the isotropic point can be found in [1]. We have little to add to this subject, except to say that the sum rules involving an external scale, such as a lattice size dependence, must be expressed with a symmetric finite-difference scheme to avoid $\mathrm{O}(a)$ discretization errors. Here is a nice application: for a torelon in the direction $\hat{1}$, the energy density in the transverse plane is directly sensitive to quantum string corrections, as already noted in [1], and an effective central charge can be defined via

$$
c_{\text {eff }}\left(L=n a, \breve{\ell}_{0}\right)=\frac{n}{2}(U-S)\left[\square_{02}+\square_{03}-\left.2 \square_{23}\right|_{\Omega} ^{n} .\right.
$$

The definition of the anisotropic derivatives $U$ and $S$ will be given in Section 3.2; we note that $(U-S) / 2=\beta-N\left(c_{\sigma}-c_{\tau}\right)$, where the coefficients $c_{\sigma}, c_{\tau}$ were determined in [17] (see also references therein) for a few $\beta$ values. The effective central charge extracted in this way will differ by $\mathrm{O}\left(a^{2}\right)$ terms from Eq. (24).

\section{Lattice sum rules with Wilson fermions}

We start by recalling some essential facts about Wilson fermions and their transfer matrix. We shall use $\mathbf{x}, \mathbf{y}$ for three-component spatial vectors, indices $\alpha, \beta, \ldots$ for color and $\rho, \sigma, \ldots$ for spinor indices. A spinor component is written as $\psi_{\sigma}(\mathbf{x}, \alpha)$; for simplicity we consider the oneflavour theory for the moment, but the extension to several flavours is trivial, as we shall see. We 
use a set of Euclidean Dirac matrices, $\left\{\gamma_{\mu}, \gamma_{\nu}\right\}=2 \delta_{\mu \nu}$, as well as the projectors $P_{ \pm}=\frac{1}{2}\left(1 \pm \gamma_{0}\right)$. Whenever there is a risk of confusion, we use a hat to distinguish a quantum mechanical operator from a Euclidean c-number or Grassmann variable. The full Hilbert space $\mathcal{H}$ is now the tensor product of the gauge Hilbert space $\mathcal{H}_{G}$ and a fermionic Hilbert space $\mathcal{H}_{F}$.

An important object is the color-covariant, nearest-neighbour transport operator for fields transforming in the fundamental representation:

$$
D_{k}^{ \pm}(\mathbf{x}, \alpha ; \mathbf{y}, \beta)=U_{k}(\mathbf{x})_{\alpha \beta} \delta_{\mathbf{x}+a \hat{k}, \mathbf{y}} \pm U_{k}^{\dagger}(\mathbf{y})_{\alpha \beta} \delta_{\mathbf{y}+a \hat{k}, \mathbf{x}}, \quad k=1,2,3 .
$$

Two spatial finite-difference operators will be used:

$$
\begin{aligned}
& B=\mathbf{1}-\kappa \sum_{k} D_{k}^{+}, \\
& C=\frac{1}{2} \sum_{k} D_{k}^{-} \otimes \gamma_{k} .
\end{aligned}
$$

For any gauge field configuration [14], $B$ is hermitian and strictly positive for $0<\kappa<1 / 6$, while $C$ is anti-Hermitian.

The fermionic Hilbert space is the Fock space built from a collection of $\hat{\chi}$ operators, which enjoy canonical anticommutation relations $\left(\left\{\hat{\chi}_{\sigma}(\mathbf{x}, \alpha), \hat{\chi}_{\sigma^{\prime}}^{\dagger}(\mathbf{y}, \beta)\right\}=\delta_{\mathbf{x y}} \delta_{\sigma \sigma^{\prime}} \delta_{\alpha \beta}\right.$ etc.). One further defines [14]

$$
\hat{\psi}_{\sigma}=B^{-1 / 2} \hat{\chi}_{\sigma}, \quad \hat{\psi}_{\sigma}^{\dagger}=\hat{\chi}_{\sigma}^{\dagger}\left(B^{-1 / 2}\right)^{t},
$$

that turn out to be the operators associated with the Grassmann variables $(\bar{\psi}, \psi)$.

The transfer matrix in the temporal gauge $A_{0}=0$ was obtained in terms of the $\hat{\chi}$ operators in $[14,15]$. The kernel of the transfer matrix in the path integral form is given in [20], Eq. (4.26):

$$
\begin{aligned}
& K\left[V, \bar{\psi}, \psi, V^{\prime}, \bar{\psi}^{\prime}, \psi^{\prime}\right] \\
& =\operatorname{det}\left\{B B^{\prime}\right\}^{1 / 2} \int \prod_{\mathbf{x}} \mathrm{d} W(\mathbf{x}) e^{-\beta \Delta S\left[V, W, V^{\prime}\right]} \\
& \quad \times \exp \left\{-2 \kappa\left(\bar{\psi} P_{+} C \psi-\bar{\psi} P_{+} W^{-1} \psi^{\prime}-\bar{\psi}^{\prime} W P_{-} \psi+\bar{\psi}^{\prime} P_{-} C^{\prime} \psi^{\prime}\right)\right\},
\end{aligned}
$$

where $B^{\prime}, C^{\prime}$ are functionals of $V^{\prime}$. The scalar product of two functionals of $(\bar{\psi}, \psi)$ is taken with respect to Grassmann integration with a measure $e^{-\bar{\psi} B \psi} / \operatorname{det} B$. The determinant arises because of the change of basis (39).

We can now derive a sum rule in the same fashion as in the pure gauge case. Let $\Phi$ be a normalized eigenstate of the transfer matrix with eigenvalue $\lambda$. We find ${ }^{1}$

$$
\begin{aligned}
\partial_{\kappa} \lambda= & \partial_{\kappa}\langle\Phi|\mathbf{T}(\beta, \kappa)| \Phi\rangle \\
= & 2 \int \mathrm{D} V \mathrm{D} \bar{\psi} \mathrm{D} \psi \operatorname{det}\{B\}^{-1 / 2} e^{-\bar{\psi} B \psi} \Phi[V, \bar{\psi}, \psi]^{*} \\
& \times \int \mathrm{D} V^{\prime} \mathrm{D} \bar{\psi}^{\prime} \mathrm{D} \psi^{\prime} \operatorname{det}\left\{B^{\prime}\right\}^{-1 / 2} e^{-\bar{\psi}^{\prime} B^{\prime} \psi^{\prime}} \Phi\left[V^{\prime}, \bar{\psi}^{\prime}, \psi^{\prime}\right]
\end{aligned}
$$

1 It may be useful to note that if $T(x, y)=T^{*}(y, x), \quad \lambda=\int w(x) \mathrm{d} x w(y) \mathrm{d} y \phi^{*}(x) T(x, y) \phi(y)$ and $\int \phi^{*}(x) \phi(x) w(x) \mathrm{d} x=1$, where $T, \phi$ and $w$ all depend on a parameter $\kappa$, then $\partial_{\kappa} \lambda=$ $\int w(x) \mathrm{d} x w(y) \mathrm{d} y \phi^{*}(x)\left(\partial_{\kappa} T(x, y)\right) \phi(y)+\lambda \int\left(\partial_{\kappa} w(x)\right) \mathrm{d} x|\phi(x)|^{2}$. 


$$
\begin{aligned}
& \times \int \mathrm{D} W e^{-\beta \Delta S\left[V, W, V^{\prime}\right]}\left(\bar{\psi} P_{+} W^{-1} \psi^{\prime}+\bar{\psi}^{\prime} W P_{-} \psi\right) \\
& \times \exp \left\{-2 \kappa\left(\bar{\psi} P_{+} C \psi-\bar{\psi} P_{+} W^{-1} \psi^{\prime}-\bar{\psi}^{\prime} W P_{-} \psi+\bar{\psi}^{\prime} P_{-} C^{\prime} \psi^{\prime}\right)\right\} \\
& +\lambda \int \mathrm{D} V \mathrm{D} \bar{\psi} \mathrm{D} \psi \frac{e^{-\bar{\psi} B \psi}}{\operatorname{det} B}|\Phi[V, \bar{\psi}, \psi]|^{2}\left(\bar{\psi}\left(\frac{1}{\kappa}(1-B)-2 C\right) \psi\right)
\end{aligned}
$$

In the last line, $\lambda$ multiplies the expectation value on the state $\Phi$ of an equal-time operator $\hat{A}_{s}$, while the rest of the expression is the expectation value on the state $\Phi$ of an integral operator $\hat{A}_{t}$, times $\lambda_{\Omega}$. Following the discussion of the pure gauge case, we thus have

$$
-\partial_{\kappa} \breve{E}(\beta, \kappa)=\left\langle\Phi\left|\hat{A}_{s}\right| \Phi\right\rangle-\left\langle\Omega\left|\hat{A}_{s}\right| \Omega\right\rangle+e^{\breve{E}}\left\langle\Phi\left|\hat{A}_{t}\right| \Phi\right\rangle-\left.\left\langle\Omega\left|\hat{A}_{t}\right| \Omega\right\rangle \equiv \mathrm{h}\right|_{\Omega} ^{\Phi} .
$$

To evaluate this matrix element in a Monte Carlo simulation, one would use an interpolating field $\varphi$ for the state $\Phi$. If it is the lightest in its symmetry channel,

$$
\left\langle\Phi\left|\hat{A}_{s}\right| \Phi\right\rangle=\lim _{x_{0} \rightarrow \infty} f_{s}\left(x_{0}\right), \quad e^{\breve{E}}\left\langle\Phi\left|\hat{A}_{t}\right| \Phi\right\rangle=\lim _{x_{0} \rightarrow \infty} f_{t}\left(x_{0}\right)
$$

with (for $x_{0} / a$ even)

$$
\begin{aligned}
f_{s}\left(x_{0}\right) & =\frac{\left\langle\bar{\varphi}(0) \frac{1}{2}\left(A_{s}\left(x_{0} / 2\right)+A_{s}\left(x_{0} / 2+a\right)\right) \bar{\varphi}\left(x_{0}+a\right)\right\rangle}{\left\langle\bar{\varphi}(0) \bar{\varphi}\left(x_{0}+a\right)\right\rangle}, \\
f_{t}\left(x_{0}\right) & =\frac{\left\langle\bar{\varphi}(0) A_{t}\left(x_{0} / 2\right) \bar{\varphi}\left(x_{0}+a\right)\right\rangle}{\left\langle\bar{\varphi}(0) \bar{\varphi}\left(x_{0}+a\right)\right\rangle}
\end{aligned}
$$

and

$$
\begin{aligned}
& A_{s}\left(x_{0}\right)=\sum_{\mathbf{x}, k} \bar{\psi}(x) U_{k}(x)\left(1-\gamma_{k}\right) \psi(x+a \hat{k})+\bar{\psi}(x+a \hat{k}) U_{k}(x)^{-1}\left(1+\gamma_{k}\right) \psi(x), \\
& A_{t}\left(x_{0}\right)=\sum_{\mathbf{x}} \bar{\psi}(x) U_{0}(x)\left(1-\gamma_{0}\right) \psi(x+a \hat{0})+\bar{\psi}(x+a \hat{0})\left(1+\gamma_{0}\right) U_{0}(x)^{-1} \psi(x) .
\end{aligned}
$$

These are the hopping terms of the Wilson-Dirac action; they are the terms one would obtain by naively differentiating the Boltzmann factor. The operators $\hat{A}_{t}$ and $\hat{A}_{s}$ have been defined in such a way that their vacuum expectation values match the Euclidean expectation values of $A_{t}$ and $A_{s}$ respectively, for periodic boundary conditions in the time direction with $L_{0} \rightarrow \infty$. It is also clear now that Eqs. (41) and (45), (46) hold for $N_{\mathrm{f}}$ degenerate flavours, provided an implicit summation over flavours is understood in (45), (46). In practice, once the fermion fields are integrated out, the three-point functions involve all-to-all propagators within one time-slice. A stochastic estimator is then required, as used in previous thermodynamics applications [24].

\subsection{Applications}

We now present some applications of the sum rule just established. We focus on the case of $N_{\mathrm{f}}$ degenerate flavours. Let us assume for now the spatial volume to be large enough for the state $\Phi$ to suffer negligible finite volume effects (for a stable one-particle state, they are exponentially small). Given the existence of the continuum limit, we have, up to cutoff effects,

$$
0=\frac{\mathrm{d}}{\mathrm{d} a}\left[\frac{\breve{E}}{a}(\beta(a), \kappa(a))\right] \Rightarrow \quad \breve{E}=\partial_{\beta} \breve{E} \frac{\mathrm{d} \beta}{\mathrm{d} \log a}+\partial_{\kappa} \breve{E} \frac{\mathrm{d} \kappa}{\mathrm{d} \log a} .
$$


But actually, $\kappa=\kappa(\beta(a))$ : on a line of 'constant physics' $\kappa$ must be tuned as a function of $\beta$. Hence

$$
\breve{E}=\frac{\mathrm{d} \beta}{\mathrm{d} \log a}\left[\square_{\Omega}^{\Phi}-\left.\frac{\mathrm{d} \kappa}{\mathrm{d} \beta} \mathrm{h}\right|_{\Omega} ^{\Phi}\right][1+\mathrm{O}(a)],
$$

where we have used Eqs. (10) and (42). Recall that $\frac{\mathrm{d} \beta}{\mathrm{d} \log a} \sim-4 N b_{0}$ is universal in the continuum limit $\left(b_{0}=\frac{1}{3(4 \pi)^{2}}\left(11 N-2 N_{\mathrm{f}}\right)\right)$. A particularly interesting case arises for $N_{\mathrm{f}} \geqslant 2$ in the chiral limit, $\kappa=\kappa_{c}$. The 'pion' mass then vanishes and hence

$$
\frac{\mathrm{d} \kappa_{c}}{\mathrm{~d} \beta}=\frac{\square_{\Omega}^{\pi}}{\left.\mathrm{h}\right|_{\Omega} ^{\pi}}[1+\mathrm{O}(a)] .
$$

Note that at no stage in the derivation of the sum rules did we need to make an assumption on the extent of the time direction - only the spatial lattice size was assumed to be essentially infinite. Hence this equation also holds in the Schrödinger functional-where one can actually simulate at $\kappa_{c}$. Relation (49) can now be inserted into the sum rule for the only other stable particle, the nucleon:

$$
\breve{M}_{\text {nucl }}=\frac{\mathrm{d} \beta}{\mathrm{d} \log a}\left[\square_{\Omega}^{\text {nucl }}-\frac{\left.\mathrm{h}\right|_{\Omega} ^{\text {nucl }}}{\left.\mathrm{h}\right|_{\Omega} ^{\pi}} \square_{\Omega}^{\pi}\right][1+\mathrm{O}(a)] .
$$

As in the pure gauge theory, we can give an improved estimate of the continuum $z \equiv \breve{\ell}_{0} \breve{M}$, assuming now that the leading corrections to the spectrum are $\mathrm{O}(a)$. Here $\breve{\ell}_{0}\left(\beta, \kappa_{c}(\beta)\right)$ is the quantity evaluated in the chiral limit; concrete examples are $\left.r_{0}\right|_{m_{\pi}=0}, \Lambda_{\overline{\mathrm{MS}}}^{-1}$ or $L_{\mathrm{max}}$, defined by $\bar{g}^{2}\left(1 / L_{\max }\right)=x$, where $x$ is a particular numerical value and $\bar{g}^{2}$ the renormalized Schrödinger functional coupling [23]. We get

$$
z_{\text {impr }}\left(\breve{\ell}_{0}\right) \equiv z\left(\breve{\ell}_{0}\right)+\frac{\mathrm{d} z}{\mathrm{~d} \log \breve{\ell}_{0}}=2 z\left(\breve{\ell}_{0}\right)-\breve{\ell}_{0} \frac{\mathrm{d} \beta}{\mathrm{d} \log a}\left(\square_{\Omega}^{\Phi}-\left.\frac{\mathrm{d} \kappa}{\mathrm{d} \beta} \mathrm{h}\right|_{\Omega} ^{\Phi}\right) .
$$

We expect the $\mathrm{O}(a)$ cutoff effects to be substantially reduced on this estimator. Note that for this formula to yield an improvement, $\frac{\mathrm{d} \beta}{\operatorname{d} \log a}$ must be defined through the quantity $\ell_{0}$, as in Eq. (14).

If the volume dependence of the energy level cannot be neglected, Eq. (48) becomes

$$
\breve{E}\left(\left\{\breve{L}_{k}\right\}\right)+\sum_{k} \breve{L}_{k} \tilde{\partial}_{\breve{L}_{k}} \breve{E}=\frac{\mathrm{d} \beta}{\mathrm{d} \log a}\left[\square-\left.\frac{\mathrm{d} \kappa}{\mathrm{d} \beta} \mathrm{h}\right|_{\Omega} ^{\Phi\left(\left\{L_{k}\right\}\right)}[1+\mathrm{O}(a)] .\right.
$$

The derivation is identical to the one for the corresponding relation in the pure gauge case. The finite-difference scheme used here in the derivative is formally irrelevant, since the sum rule holds with $\mathrm{O}(a)$ corrections anyhow; however we still expect the symmetric scheme to yield somewhat smaller discretization errors. This formula may be useful to study the volume dependence of the pion mass, and also of the state which is a mixture of a two-pion state and the $\rho$ resonance. The latter volume dependence allows one to extract the scattering lengths of pions in the elastic regime and the $\rho$ width [22].

\subsection{Anisotropic lattice}

We consider the Wilson theory with $N_{\mathrm{f}}$ degenerate flavours. If one assigns an independent hopping parameter in the space directions from the time direction, then one can derive the equa- 
tions

$$
\begin{gathered}
-\frac{\partial \breve{E}}{\partial \kappa_{\tau}}=e^{\breve{E}}\left\langle\Phi\left|\hat{A}_{t}\right| \Phi\right\rangle-\left.\left\langle\Omega\left|\hat{A}_{t}\right| \Omega\right\rangle \equiv \mathrm{h}_{t}\right|_{\Omega} ^{\Phi}, \\
-\frac{\partial \breve{E}}{\partial \kappa_{\sigma}}=\left\langle\Phi\left|\hat{A}_{s}\right| \Phi\right\rangle-\left.\left\langle\Omega\left|\hat{A}_{s}\right| \Omega\right\rangle \equiv \mathrm{h}_{s}\right|_{\Omega} ^{\Phi} .
\end{gathered}
$$

To exploit these relations in a useful way, we must understand how the theory with parameters $\left(\beta_{s}, \beta_{t}, \kappa_{s}, \kappa_{t}\right)$ is renormalized. As independent 'physical' variables, the most intuitive choice is $\left(\breve{\ell}_{0}, \xi, x\right)$, where $\xi$ is the anisotropy $a_{s} / a_{t}$ and $x$ essentially sets the quark masses (e.g. $x=$ $\left.m_{\mathrm{PS}} / m_{\mathrm{V}}\right)$. The continuum limit is then taken with $\breve{\ell}_{0}^{-1} \rightarrow 0$ at fixed $\xi$ and $x$. One can alternatively choose the set $\left(a_{t}, a_{s}, x\right)$ as independent variables. Since there are four lattice parameters, it is only on a hypersurface in that parameter space that the lattice theory describes QCD at all. The continuum limit corresponds to a particular curve on that hypersurface where $\xi$ and $x$ are constant.

Imagine momentarily using four independent lattice spacings, so that $\left(a_{0}, a_{1}, a_{2}, a_{3}, x\right)$ are a set of independent variables. Introduce one hopping parameter $\kappa_{\mu}$ for each direction, $\beta_{\mu \nu}$ for each plaquette orientation and let $G \equiv \partial \kappa_{0} / \partial \log a_{0}$ and $H \equiv \partial \kappa_{0} / \partial \log a_{k}$. Now locking $a_{1}=$ $a_{2}=a_{3}=a_{s}$, the following relations hold at the isotropic point $a_{s}=a_{t}$ :

$$
\begin{array}{ll}
\partial \kappa_{k} / \partial a_{t}=H, & \partial \kappa_{k} / \partial a_{s}=G+2 H, \\
\partial \kappa_{0} / \partial a_{t}=G, & \partial \kappa_{0} / \partial a_{s}=3 H .
\end{array}
$$

Similarly, if $S=\partial \beta_{0 k} / \partial \log a_{0}$ and $U \equiv \partial \beta_{k l} / \partial \log a_{0}$, then in the $a_{1}=a_{2}=a_{3}=a_{s}$ theory, we have at the isotropic point $a_{s}=a_{t}[1]$ :

$$
\begin{array}{ll}
\partial \beta_{0 k} / \partial \log a_{t}=S, & \partial \beta_{0 k} / \partial \log a_{s}=S+2 U, \\
\partial \beta_{k l} / \partial \log a_{t}=U, & \partial \beta_{k l} / \partial \log a_{s}=2 S+U .
\end{array}
$$

In this way the number of functions has been reduced by a factor two. However, to our knowledge the derivatives $U, S, G, H$ have not yet been determined individually for $N_{\mathrm{f}} \geqslant 2$.

By writing the renormalization group equations $\frac{\mathrm{d}}{\mathrm{d} a_{s, t}} \frac{\breve{E}}{a_{t}}=0$, and taking suitable linear combinations thereof, one recovers Eq. (48) (or more generally Eq. (52)) under the consistency conditions

$$
2(S+U)=\frac{\mathrm{d} \beta}{\mathrm{d} \log a}, \quad G+3 H=\frac{\mathrm{d} \kappa}{\mathrm{d} \beta},
$$

and obtains the independent sum rule

$$
\begin{aligned}
\breve{E} & -\frac{1}{3} \sum_{k} \breve{L}_{k} \tilde{\partial}_{\breve{L}_{k}} \breve{E} \\
& =-\left\{\frac { 2 } { 3 } ( U - S ) \left[\sum_{k} \square_{0 k}-\left.\sum_{k<l} \square_{k l}\right|_{\Omega} ^{\Phi}+\frac{1}{3}(G-H)\left[3 \mathrm{~h}_{t}-\left.\mathrm{h}_{s}\right|_{\Omega} ^{\Phi}\right\}[1+\mathrm{O}(a)] .\right.\right.
\end{aligned}
$$

Here the vacuum matrix elements vanish in the $L_{k} \rightarrow \infty$ limit by Euclidean symmetry.

In thermodynamic studies of QCD using the Wilson regularization [24], the energy density $\epsilon$ and the pressure $p$ are extracted in the so-called derivative method by computing derivatives with respect to $\beta$ and $\kappa$ of the partition function. Since $\mathcal{Z}=\operatorname{Tr}\left\{\mathbf{T}^{\breve{L}_{0}}\right\}$, where the temperature $T$ is 
equal to $L_{0}^{-1}$, one finds that $a V(\epsilon-3 p)$, the 'interaction measure', is given by a thermal average (with Boltzmann measure $e^{-E / T}$ ) over the eigenstates of the transfer matrix of expression (52). Similarly the expression corresponding to $a V(\epsilon+p)$ is Eq. (58).

\section{Conclusion}

We have extended the known set of lattice sum rules by including the effects of Wilson fermions. Our derivation in the transfer matrix formalism kept track of discretization errors. The main results are Eqs. (52) and (58).

We have also presented new applications of the sum rules. Since they allow us to compute the derivative of an energy level with respect to the lattice spacing, we have proposed to exploit this to reduce cutoff effects on the spectrum and on the static potential. One may include the information on the slope in the continuum extrapolation, if one disposes of data at several lattice spacings. Alternatively, one can form an improved estimate of the continuum limit from data at a single lattice spacing under the assumption of a functional form for the leading discretization errors, such as the one predicted by Symanzik's effective theory [21].

Of course these applications are only of practical interest if the three-point functions can be computed accurately in numerical simulations. This can probably be achieved for the static potential in the pure gauge theory using a suitable multi-level algorithm [25]. It also seems realistic in $N_{\mathrm{f}} \geqslant 2$ theories in the pseudoscalar sector, although high statistics and efficient all-to-all techniques will be needed. We remark that higher order sum rules may be derived straightforwardly by taking additional derivatives of the transfer matrix with respect to the bare parameters, but the $n$-point functions are bound to become more difficult to evaluate numerically with increasing $n$. It is nevertheless conceptually pleasing that locally the lattice artifacts can be determined at fixed bare lattice parameters.

\section{Acknowledgements}

I am happy to thank Karl Jansen and Rainer Sommer for reading and commenting on the manuscript.

\section{References}

[1] C. Michael, Phys. Rev. D 53 (1996) 4102, hep-lat/9504016.

[2] H.J. Rothe, Phys. Lett. B 355 (1995) 260, hep-lat/9504012.

[3] C. Michael, Nucl. Phys. B 280 (1987) 13.

[4] G.A. Tickle, C. Michael, Nucl. Phys. B 333 (1990) 593.

[5] A.M. Green, C. Michael, P.S. Spencer, Phys. Rev. D 55 (1997) 1216, hep-lat/9610011.

[6] C. Michael, A.M. Green, P.S. Spencer, Phys. Lett. B 386 (1996) 269, hep-lat/9606002.

[7] G.S. Bali, C. Schlichter, K. Schilling, Phys. Lett. B 363 (1995) 196, hep-lat/9508027.

[8] H.J. Rothe, Phys. Lett. B 364 (1995) 227, hep-lat/9508005.

[9] M. Lüscher, hep-lat/9802029.

[10] M. Lüscher, S. Sint, R. Sommer, P. Weisz, Nucl. Phys. B 478 (1996) 365, hep-lat/9605038;

M. Lüscher, S. Sint, R. Sommer, P. Weisz, U. Wolff, Nucl. Phys. B 491 (1997) 323, hep-lat/9609035.

[11] M. Lüscher, PoS LAT2005 (2006) 002, hep-lat/0509152.

[12] C. Alexandrou, G. Koutsou, J.W. Negele, A. Tsapalis, Phys. Rev. D 74 (2006) 034508, hep-lat/0605017; C. Alexandrou, T. Leontiou, J.W. Negele, A. Tsapalis, hep-lat/0607030.

[13] M. Lüscher, DESY-88-156, Lectures given at Summer School 'Fields, Strings and Critical Phenomena', Les Houches, France, 28 June-5 August 1988.

[14] M. Lüscher, Commun. Math. Phys. 54 (1977) 283. 
[15] M. Creutz, Phys. Rev. D 15 (1977) 1128.

[16] M. Lüscher, P. Weisz, JHEP 0207 (2002) 049, hep-lat/0207003.

[17] J. Engels, F. Karsch, T. Scheideler, Nucl. Phys. B 564 (2000) 303, hep-lat/9905002.

[18] S. Necco, R. Sommer, Nucl. Phys. B 622 (2002) 328, hep-lat/0108008.

[19] R. Sommer, Nucl. Phys. B 411 (1994) 839, hep-lat/9310022.

[20] S. Sint, Nucl. Phys. B 421 (1994) 135, hep-lat/9312079.

[21] K. Symanzik, Nucl. Phys. B 226 (1983) 187;

K. Symanzik, Nucl. Phys. B 226 (1983) 205.

[22] M. Lüscher, Nucl. Phys. B 364 (1991) 237.

[23] ALPHA Collaboration, M. Della Morte, R. Frezzotti, J. Heitger, J. Rolf, R. Sommer, U. Wolff, Nucl. Phys. B 713 (2005) 378, hep-lat/0411025.

[24] CP-PACS Collaboration, A. Ali Khan, et al., Phys. Rev. D 64 (2001) 074510, hep-lat/0103028.

[25] H.B. Meyer, in preparation. 